\title{
Relationship between ureterovesical flow and the size of ureteral lites measured with doppler ultrasound
}

\begin{abstract}
Summary
Background: The prevalence of lithiasis in the urinary tract is estimated at $12 \%$ of the general population in industrialized countries, of which $2.3 \%$ of the population that suffers it with a picture of renal colic. Radiological studies have been the tool used to establish the diagnosis of this pathology. The Gold Standard is computed tomography because it has a sensitivity of $96 \%-98 \%$ and a specificity of $95 \%-98 \%$. We currently know that Ultrasound is a first-line tool and with technological advances has allowed us to identify almost all of this pathology, visualizing the renal anatomical alterations, ureters and the difference between the pathological and non-pathological bladder ureter jet.
\end{abstract}

Material and methods: Analytical study, observations, prospective and correlation. The sample consists of patients older than 18 years who enter the emergency department of the Regional Hospital of Monterrey with the diagnosis of ureteral lithiasis, which has been performed Doppler Ultrasound and Computed Tomography, with entering the department of urology in the study period, and that meet the inclusion criteria in the period from March 2018 to November 2018

Results: Of the patients found, the majority were women, with 16 samples having an average of $72.7 \%$ of the general population. An almost similar relationship was found in the distribution of affected ureters, being mainly the right ureter.

Discussion and conclusion: The use of Doppler ultrasound for the detection and measurement of Ureterovesical flow proved to be an effective method to correlate the size of the stone when it occurs in the uretero. Ultrasound has been placed as a form of current diagnosis for ureteral lithiasis, contrary to what was previously believed because the BMI, age and other factors affect the image at the time of diagnosing the lithium, but new forms have been found to relate this pathology as they are through the degree of hydronephrosis. It was shown that the larger the size of the stone, the lower the ureterovesical flow and vice versa the smaller the size of the stone, the greater the ureterovesical flow.

Keywords: urolithiasis, doppler ultrasound, hydronephrosis, calculi
Volume 3 Issue 6 - 2019

\author{
Rolando Martínez-Hernández,' Paul \\ Montoya-Alarcón ${ }^{2}$ \\ 'Emergency Department, ISSSTE Regional Hostipal Monterrey, \\ Graduate from Universidad de Monterrey, México \\ ${ }^{2}$ Medstudent at intership year, ISSSTE Regional Hostipal \\ Monterrey, Student at Universidad de Monterrey, México
}

Correspondence: Rolando Martínez Hernández, Emergency Department, ISSSTE Regional Hostipal Monterrey, Graduate from Universidad de Monterrey, Monterrey, Nuevo León, México,Tel 8121820817,Email Rmtz06@gmail.com

Received: August 19,2019 | Published: November 07, 2019

\section{Introduction}

Ureteral flow is defined as the flow of urine that passes through the ureterovesical orifice into the bladder. ${ }^{1,2}$ The prevalence of calculus in the urinary tract is estimated at $12 \%$ of the general population in industrialized countries, of which $2.3 \%$ of the population that suffers from it have a renal colic disorder. ${ }^{2-6}$ Radiological studies have been the tool used to establish the diagnosis of this pathology, although we know simple abdominal radiography has shown a low sensitivity and specificity to diagnose this pathology, ${ }^{7}$ we know that the gold standard is computed tomography because since it has a sensitivity of $96 \%$ - 98\% and a specificity of $95 \%-98 \%$, nowadays the low-dose computed tomography (1.5-5 millisiverts) is the best way to detect the urinary tract stones. ${ }^{8-10}$ We know that the CT scan has disadvantages such as the high cost to carry them out and the high exposure to radiation. ${ }^{11,12}$ We currently know that Ultrasound is a first-line tool for the study of this pathology, since it has a sensitivity of $40 \%-90 \%$ and a specificity of $79 \%-100 \% .{ }^{10-16}$ But with the technological advances in this ultrasound area has allowed us to identify almost completely said pathology, visualizing renal anatomical alterations, ureters and the difference between the pathological and non-pathological bladder ureter jet. ${ }^{17-24}$

This protocol aims to study the existence of a correlation between the size of the ureteral calculus and the bladder ureter jet, because as mentioned above, a distortion in this flow has been reported when this pathology occurs.Technological and methodological advances in the field of radiology have allowed us to diagnose ureteral stones with great certainty by means of Computed Tomography as a gold standard, and by ultrasound as a first-line diagnosis, but sometimes there is no means to be able to make the diagnosis in certain units since they do not have CT, we believe that the definitive diagnosis of this disease can be made and its size can be calculated only with simple use of ultrasound and its derived methods such as Doppler.

\section{Materials and methods}

Analytical study, observations, prospective and correlation. The sample consists of patients over 18 years old who enter through the 
emergency department of the ISSSTE Monterrey Regional Hospital with the diagnosis of ureteral lithiasis, who have undergone Doppler Ultrasound and Computed Tomography, who enter the urology department during the study period, and that meet the inclusion criteria in the period from March 2018 to November 2018.

\section{Inclusion criteria}

I. Right patients of the ISSSTE

II. Have a Doppler Ultrasound and CT Scan

III. Be over 18 years old.

\section{Exclusion criteria}

I. Patients with renal or bladder stones

II. Patients presenting with malformations of the genitourinary tract

III. Obese or chronic kidney disease patients who are contraindicated to perform CT

IV. Patients who have previous Double J placement surgery.

\section{Elimination criteria}

I. Decision to exit the investigation protocol

II. Patients whose diagnosis of admission is ureteral lithiasis, but at the time of performing the Ultrasound and Computed Tomography test the calculus is outside the anatomical area mentioned above.

Doppler Ultrasound Technique: The patient is hydrated orally with $1000 \mathrm{ml}$ of water. The reno-bladder study was performed with a PROSOUND ALPHA6 ultrasound using the convex transducer, using 3.5 MHz. Bladder volume, parenchymal thickness and hydronephrosis grade were evaluated by grayscale. When presenting a bladder with a volume of $110 \mathrm{ml}$, at least 15 minutes of continuous real time should be observed and the holes in the axial plane were observed and recorded simultaneously by means of color Doppler ultrasound. The red flow system assigned to the flow direction that approaches the transducer. From then on, the measurements of the ureterovesical flow were recorded in centimeters over a second.

Urinary Tract Computed Tomography Technique: Cuts of the images of UroTac with Split bolus technique were taken, to patients who previously had an ultrasound. Once at the tomograph table, with the patient supine, a Scouty is performed an acquisition without contrast from the T12 vertebral level up to $2 \mathrm{~cm}$ below the pubic symphysis. With a reconstruction of the cuts of 1-2 mm, taking axial, sagittal and coronal measurements, recording the length of the three cuts mentioned above. Subsequently, it will be saved in the "DICOM Viewer" system and then re-evaluated.

\section{Ethical aspect}

The present study is considered low risk since it does not threaten the human or social integrity of patients or their families and is within the margins stipulated in the Regulations of the General Health Law on Health Research, Regulation of Biomedical research by the Mexican Health Code article 1-14, as well as all international laws and agreements such as the Declaration of Helsinki and subsequent amendments. Because it is considered without risk, an informed consent letter is not required. The confidentiality of the information obtained is guaranteed so that work will be carried out with identification of the cases by means of designation of numerical code. The anonymity of patients will be maintained in sessions, congresses, publications and manuscripts that are generated from the study, always respecting their identity.

\section{Results}

They were 23 patients who met the inclusion criteria. In total, data were collected from 25 patients; however 2 were cataloged with exclusion criteria because they could not have a CT scan. All the patients admitted by the emergency department over 18 years of age were captured, of which the youngest patient was 24 years old and the oldest one was 81 years old, presenting an average of 38 years. Of the patients found, the majority were women (16 patients), having an average of $72.7 \%$ of the general population (Table 1). The presence of ureterovesical flow was found by Doppler ultrasound in 17 patients having an average of $77.3 \%$, the rest of the patients could not detect the ureterovesical jet, of which the smallest calculus was 4.9 MM, and the largest it was measured at $12 \mathrm{MM}$ (Table 2). An almost similar relationship was found in the distribution of the affected ureters, mainly the right ureter with a total of 12 patients, presenting a percentage of $54.5 \%$ of all registered patients. In this study, no patients were found to have both affected ureters (Table 3). As a final result, using a variable correlation plot by drawing a line from which it goes from the upper left to the lower right. This graph gives us a directly proportional relationship between the size of the calculus and the ureterovesical flow present measured by USG Doppler, of which the data found on the right side expresses a larger calculus which are positioned at the bottom of the graphic, which means that it has a lower bladder urethral flow, as the data on the size of the calculus are shifted to the left of the graph, the bladder urethral flow score is in a higher position which expresses that the smaller the size of the calculus is greater ureterovesical flow will present (Figure 1).

Table I Indicate the frequency by sex

\begin{tabular}{llll}
\hline Gender & & & \\
\hline Men & & Frequency & Percentage \\
Women & 16 & 27.3 & $27.30 \%$ \\
& 22 & 100 & $72.70 \%$ \\
& & $100 \%$ \\
\hline
\end{tabular}

Table 2 Indicates the presence of the flow

\begin{tabular}{lll}
\hline \multicolumn{2}{l}{ Affected ureter } \\
\hline & Frequency & Porctage \\
\hline Right & 12 & $54.50 \%$ \\
Left & 10 & $45.50 \%$ \\
& 22 & $100 \%$ \\
\hline
\end{tabular}

Table 3 Indicates the ureter that was most frequently affected

\begin{tabular}{lll}
\hline \multicolumn{3}{l}{ Affected ureter } \\
\hline & Frequency & Porctage \\
\hline Right & 12 & $54.50 \%$ \\
Left & 10 & $45.50 \%$ \\
& 22 & $100 \%$ \\
\hline
\end{tabular}




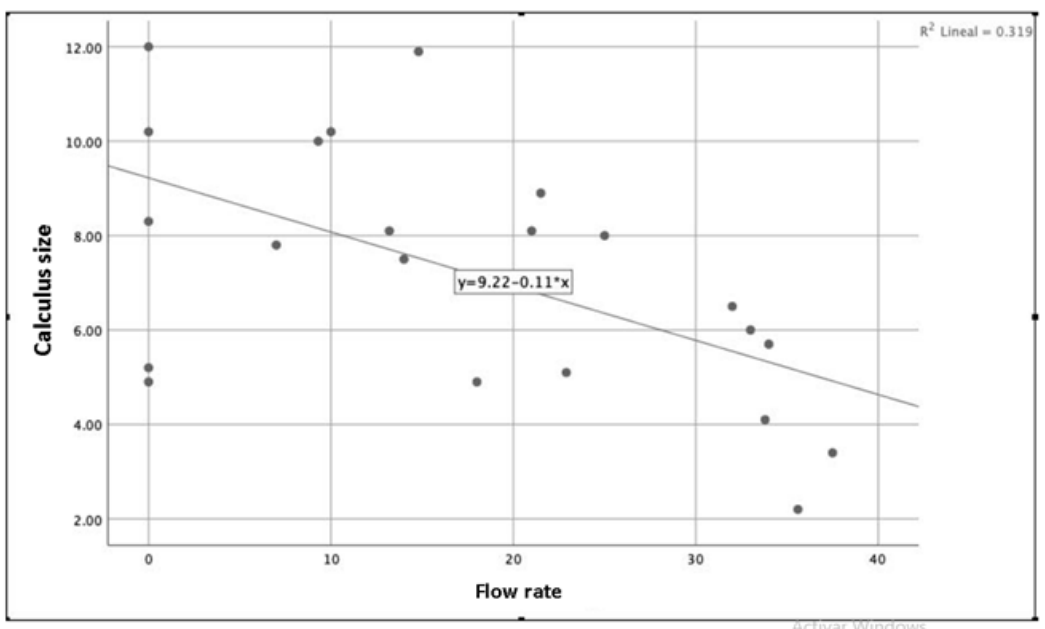

Figure I Indicates the inversely proportional relationship between the size of the coastline and the presence of the flow. The larger the size of the coastline, the lower the presence of flow.

\section{Discussion}

The use of Doppler Ultrasound for the detection and measurement of Ureterovesical flow proved to be an effective method to correlate the size of the calculus when it occurs in the uretero.The European Urology Association mentions that CT scan has positioned itself as the gold standard for the diagnosis of this pathology since it is the only imaging method capable of fully visualizing the structure of the ureter, while ultrasound has become First-line diagnosis because it is difficult to visualize the middle portion that is where most of the calculi are, because the image is interrupted by the presence of intestinal gas. Ultrasound has been placed as a current diagnostic form for ureteral lithiasis, contrary to what was previously believed because the BMI and age among other factors, affect the image at the time of diagnosing the calculus, but new ways have been found to relate this pathology as they are through the degree of hydronephrosis which does not speak of a total obstruction or almost entirely causing very little flow to pass through the light. Another way in which we relate it is through the pain present in renal colic and the distortion of the ureterovesical flow of the affected and non-pathological ureter.In the present case, all the patients who were admitted to the emergency department due to a probable diagnosis of ureteral lithiasis confirmed their diagnosis, though. The literature tells us that there is no prevalence in age and sex, but it could be found that the pathology occurs more in women than in men and the age at which it occurs most is the 4 and 5 decade of life, being the right ureter the most affected.

\section{Conclusion}

We can conclude through this study that urolithiasis is a disease that has no priority in the presentation since it can appear in the same way in men and women and at any age. It was shown that the larger the size of the calculus, the smaller the ureterovesical flow and vice versa the smaller the size of the calculus, the greater the ureterovesical flow.

\section{Acknowledgments}

None.

\section{Funding details}

None

\section{Conflicts of interest}

The author declares there is no conflict of interest.

\section{References}

1. Kanno T, Kubota M, Sakamoto $\mathrm{H}$, et al. Determining the efficacy of ultrasonography for the detection of ureteral stone. Urology. 2014;84(3):533-537.

2. Skorecki K. Brenner \& Rector's The Kidney.10 ${ }^{\text {th }}$ edn. Philadelphia: ELSEVIEER; 2016.

3. Sternberg KM, Eisner B, Larson T, et al. Ultrasonography Significantly Overestimates Stone Size When Compared to Low-dose, Noncontrast Computed Tomography. Urology. 2016;95:67-71.

4. Olympia R. Urgent Care Medicine Secrets. Philadelphia: $1^{\text {st }}$ edn. ELSEVIER; 2018.

5. Adam A. Grainger \& Allison `s Diagnostic Radiology. $6^{\text {th }}$ edn. Churchill Livingstone Elsevier; 2015.

6. Wein A. Campbell-Walsh Urology. $11^{\text {th }}$ edn. Philadelphia: ELSEVIER. 2016.

7. Winkel RR, Kalhauge A, Fredfeldt KE. The Usefulness of Ultrasound Colour-Doppler Twinkling Artefact for Detecting Urolithiasis Compared with Low Dose Nonenhanced Computerized Tomography. Ultrasound in Medicine and Biology. 2012;38(7):1180-1187.

8. Jandaghi AB, Falahatkar S, Alizadeh A, et al. Assessment of ureterovesical jet dynamics in obstructed ureter by urinary stone with color Doppler and duplex Doppler examinations. Urolithiasis. 2013;41(2):159-163.

9. Hagen-Ansert S. Textbook of diagnostic sonography. $8^{\text {th }}$ edn. St. Louis: ELSEVIER. 2018.

10. Sandhu M. Urolithiasis: Comparison of diagnostic performance of digital tomosynthesis and ultrasound. Which one to choose and when?. EJRAD. 2018; 105:25-31. 
11. Pokrajac, N. Computed tomography imaging and risk factors for clinically important diagnoses in patients presenting with flank pain. J Ermer Med. 2016;52(1):98-100.

12. Goldstone, A. Does diagnosis change as a result of repeat renal colic computed tomography scan in patients with a history of kidney stones?. Am J Emerg Med. 2010;28(3):291-295.

13. Ongun S, Teken A, Yilmaz O, et al. Relationship Between Uretera Jet Flow, Visual Analogue Scale, and Ureteral Stone Size. Urology. 2017;104:36-39.

14. Mills, L. Ultrasound for the diagnosis and management of suspected urolithiasis in the emergency department. J Emer Med. 2018;54(2):215220.

15. Camell J. Ultrasound detection of obstructive pyelonephritis due to urolithiasis in the ED. Am J Emerg Med. 2011;29(7):843.E1-843.E3.

16. Adams J. Emergency Medicine. $2^{\text {th }}$ edn. Philadelphia: ELSEVIER. 2013.

17. Celik S, Altay C, Bozkurt O, et al. Association between ureteral jet dynamics and nonobstructive kidney stones: A prospective-controlled study. Urology. 2014;84 (5):1016-1020.
18. Passerotti C, Chow JS, Silva A, et al. Ultrasound versus computerized tomography for evaluating urolithiasis. Journal of Urology. 2009;182(Suppl 4):1829-1834.

19. Ripollés T, Martínez-Pérez MJ, Vizuete J, et al. Sonographic diagnosis of symptomatic ureteral calculi: usefulness of the twinkling artifact. Abdom Imaging. 2013;38(4):863-869.

20. Fred J. Renal Imaging: Core Curriculum 2019. AJKD. 2019;73(4):552565.

21. Ng, C. Avoiding computed tomography scans by using point-of-care ultrasound when evaluating suspected pediatric renal colic. J Emer Med. 2015;49(2):165-171.

22. Oray N. A potential pitfall in the diagnosis of renal colic: renal infarction. Am J Emerg Med. 2009;27(6):750.E7-750.E9.

23. Jakubowski J. Imaging Modalities in Genitourinary Emergencies. Emerg Med Clin North Am. 2019;37(4):785-809.

24. Bhargava P. Multimodality Imaging of Ureteric Disease. Radiol Clin North Am. 2012;50(2):271-299. 